\title{
Relaxant effect of BAY 41-2272 and BAY 58-2667 in the gastrointestinal tract of apo-sGC mice
}

\author{
Sarah Cosyns ${ }^{1 *}$, Johannes-Peter Stasch² ${ }^{2}$ Peter Brouckaert ${ }^{3}$, Romain A Lefebvre ${ }^{1}$ \\ From 5th International Conference on cGMP: Generators, Effectors and Therapeutic Implications \\ Halle, Germany. 24-26 June 2011
}

\begin{abstract}
Background
In vascular tissue, relaxation by the NO-independent but heme-dependent sGC stimulator BAY 41-2272 is reduced when $\mathrm{SGC}$ is oxidized and finally loses its heme group, while that by the NO- and heme-independent sGC activator BAY 58-2667 (cinaciguat) is increased. Whether this also applies in the gastrointestinal tract has not been investigated. In apo-sGC mice, his at position 105 of sGC $\beta 1$ is mutated to phe; both sGC isoforms ( $\alpha 1 \beta 1$ and $\alpha 2 \beta 1)$ are heme-deficient and can no longer be activated by NO; this can be considered as a model for oxidized/heme-free sGC. The relaxant effect of BAY 41-2272 and BAY 58-2667 was therefore studied in the gastric fundus and distal colon of apo-sGC mice.
\end{abstract}

\section{Methods}

Homozygous apo-sGC mice and wild type (WT) controls were derived from a heterozygous breeding (mixed 129/SvJ-C57BL/6J, 11-15 weeks). Circular smooth muscle strips of the gastric fundus and distal colon (after removal of the mucosa) were mounted in Krebs solution (NANC conditions) and incubated with the sGC inhibitor ODQ $(10 \mu \mathrm{M})$ or its solvent for $30 \mathrm{~min}$. Strips were then pre-contracted with $\mathrm{PGF}_{2 \alpha}$ and the relaxant effect of cumulatively administered BAY 41-2272 (0.01-10 $\mu \mathrm{M})$ and BAY 58-2667 (1-100 nM) was examined.

\section{Results}

BAY 41-2272 (from $1 \mu \mathrm{M}$ on) and BAY 58-2667 (from $1 \mathrm{nM}$ on in the fundus and from $10 \mathrm{nM}$ on in the colon) induced a sustained relaxing response. In WT fundus strips, ODQ reduced the relaxant effect of BAY 41-2272. The relaxing effect of BAY 41-2272 was decreased in fundus strips of apo-sGC mice, but it was not influenced by ODQ. ODQ increased the relaxant effect of BAY 58-2667 in WT fundus strips. The relaxant effect of BAY 58-2667 was more pronounced in fundus strips of apo-sGC mice, but it was not influenced by ODQ. The relaxing effect of BAY 41-2272 and BAY 58-2667 did not significantly differ between colon strips of WT and apo-sGC mice. In both WT and apo-sGC colon strips, ODQ had no influence on the relaxing effect of BAY 41-2272 and BAY 58-2667.

\section{Conclusion}

At the level of the gastric fundus, BAY 58-2667 is more efficient when sGC is in the heme-free condition. At the level of the distal colon, the relaxing effect of BAY 412272 and BAY 58-2667 might to a large extent be independent of sGC stimulation/activation.

\footnotetext{
Author details

${ }^{1}$ Heymans Institute of Pharmacology, Ghent University, Ghent, Belgium.

${ }^{2}$ Cardiovascular Research, Bayer HealthCare, Wuppertal, Germany.

${ }^{3}$ Department of Molecular Biomedical Research, VIB, Ghent, Belgium.

Published: 1 August 2011
}

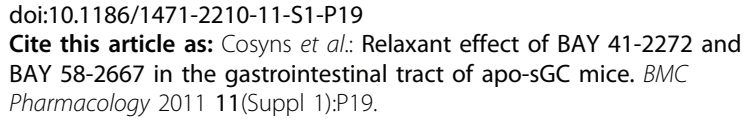

* Correspondence: sarah.cosyns@ugent.be

${ }^{1}$ Heymans Institute of Pharmacology, Ghent University, Ghent, Belgium

Full list of author information is available at the end of the article (c) 2011 Cosyns et al; licensee BioMed Central Ltd. This is an open access article distributed under the terms of the Creative Commons Attribution License (http://creativecommons.org/licenses/by/2.0), which permits unrestricted use, distribution, and reproduction in any medium, provided the original work is properly cited. 